\title{
Turismo y medio ambiente Los residuos sólidos y sus efectos en la región Cusco
}

\author{
Arístides Sotomayor Cabrera \\ Ingeniería Industrial n. 26, 2008, ISSN 1025-9929, pp. 71-81
}

Resumen: Se estima que el 50\% del total de residuos sólidos generados en la cuenca del Vilcanota, en la región Cusco, son vertidos en las márgenes de los ríos, carreteras, canales de agua y chacras, entre otros. De esta forma, el vertido incontrolado de los residuos sólidos ocasiona serios impactos ambientales, pues contaminan el agua, el suelo, afectan la salud de los pobladores y provocan el deterioro de los ecosistemas naturales, poniendo en grave riesgo el aprovechamiento de los atributos turísticos de la región. El Valle Sagrado de los Incas tiene una gran afluencia de turistas debido a los importantes monumentos arqueológicos que se encuentran en la zona. Entre los años 2001 y 2007 se ha registrado un incremento de visitantes del 80\% (420.871 y 737.576, respectivamente). En el ámbito nacional se dio similar situación, pues el número de turistas pasó de 997.628 el 2002 a 1.812 .384 el 2007.

Palabras clave: Residuos sólidos, contaminación, medio ambiente y turismo

\section{Tourism and environment: \\ Solid residues and their effects in the Cusco region}

Abstract: It is estimated that $50 \%$ of total solid residues generated in the Vilcanota river basin of the Cusco region are simply disposed in river shores, roads, water channels and small farms. This uncontrolled residue disposal in the Vilcanota valley causes serious environmental impacts which affect especially water, soil and health quality and, in general, the deterioration of natural ecosystems, putting in high risk the touristic attributes of this region. The so called Holy Valley of the Incas is a great tourist destination due to important archeological sites. It shows an 80\% increase between 2001 (420.871 visitors) and 2007 (737.576 visitors). Similarly, the total number of visitors at country level grew from 997.628 in 2002 to 1.812 .834 in 2007 .

Keywords: Solids residues, pollution, environment, tourism 


\section{RESIDUOS SÓLIDOS EN LA CUENCA DEL VILCANOTA}

El departamento de Cusco es la capital arqueológica del Perú. Allí se encuentra Machu Picchu, una de las siete nuevas maravillas del mundo, que es, asimismo, el principal destino turístico de América Latina, donde concurren anualmente cerca de un millón 400 mil turistas (Mincetur, 2007).

La generación de residuos sólidos en el departamento del Cusco, incluyendo el aporte de los turistas, es de alrededor de 380 toneladas por día (t/d), siendo la producción per cápita promedio en el valle de Vilcanota de 0,60 kg/hab.-día, cuya distribución porcentual según su composición representa más del $60 \%$ en lo que se refiere a la materia orgánica, como se puede observar en el gráfico 1.

\section{Gráfico 1 \\ Distribución de la composición de residuos sólidos}

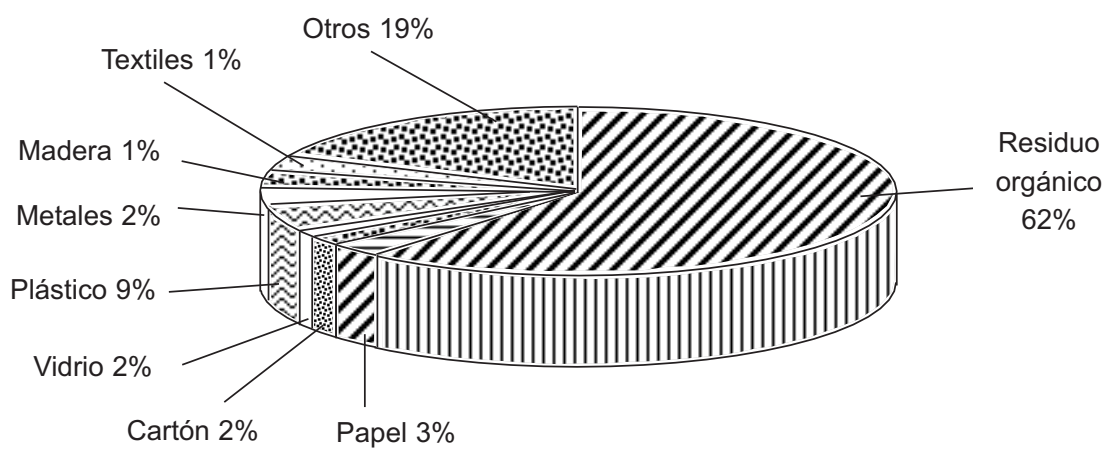

Fuente: Imaitec Consultores.

La disposición final de los residuos, en el caso de la ciudad del Cusco, se realiza en el relleno sanitario de Jaquira (fotografía 1), que está ubicado a unos 8 kilómetros de esta. Por otro lado, el relleno sanitario de Yuncachahuayco, en Urubamba, ha sido clausurado por la oposición de los comuneros de Paucarbamba, debido a la alta contaminación sobre los cultivos agrarios. Igualmente, la planta de tratamiento de compostaje en Collpani, para los residuos orgánicos generados tan- 
to por el santuario histórico de Machu Picchu como por Aguas Calientes (Machu Picchu pueblo), ha dejado de operar por oposición de los pobladores de Santa Teresa (distrito de la provincia de La Convención), debido a su inadecuada ubicación y la generación de malos olores que afectan un centro educativo cercano.

Machu Picchu y Ollantaytambo, por su parte, depositan los residuos sólidos en una cantera a unos 6 kilómetros de distancia de este último. Cabe indicar que los residuos de Aguas Calientes son trasladados por vía férrea hasta la estación de Pachar, en Urubamba, y los residuos inorgánicos son trasladados a la planta de segregación de la Municipalidad de Machu Picchu para su clasificación y reacondicionamiento, y posterior transporte en tren hasta el Cusco.

\section{Fotografía 1}

Relleno sanitario de Jaquira, Cusco

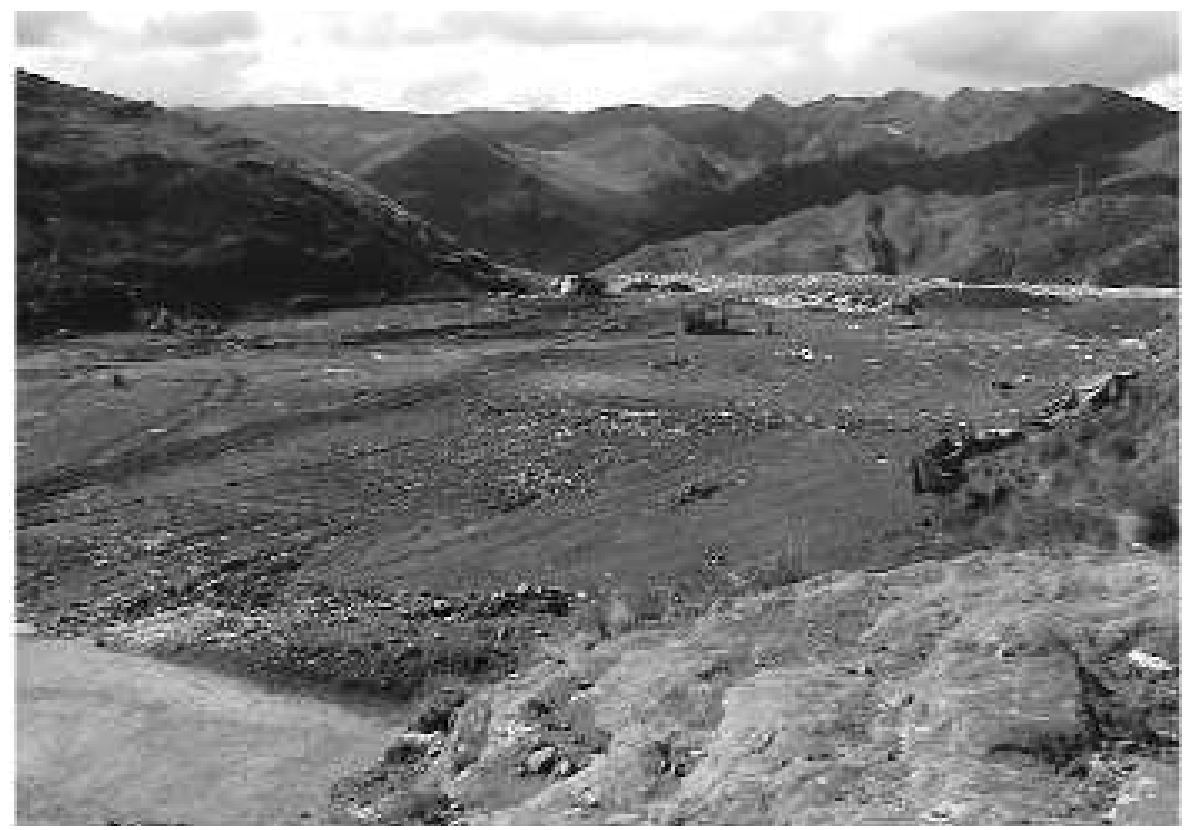

Elaboración propia. 
Por otro lado, el Servicio de Limpieza Pública (Selip) de la Municipalidad del Cusco dispone de una planta de segregación de residuos sólidos, como se puede observar en la fotografía 2. En esta se clasifican y recuperan el plástico, el papel y el cartón, además de vidrios y metales, entre otros, para su comercialización y reciclado posterior. La materia orgánica es transportada al lugar denominado Rayallacta, que está ubicada en el distrito de Andahuayllillas, para su conversión en compostaje (abono orgánico), que se utiliza en la agricultura en el valle de Urubamba.

\section{Fotografía 2}

Planta de segregación de Selip, Cusco

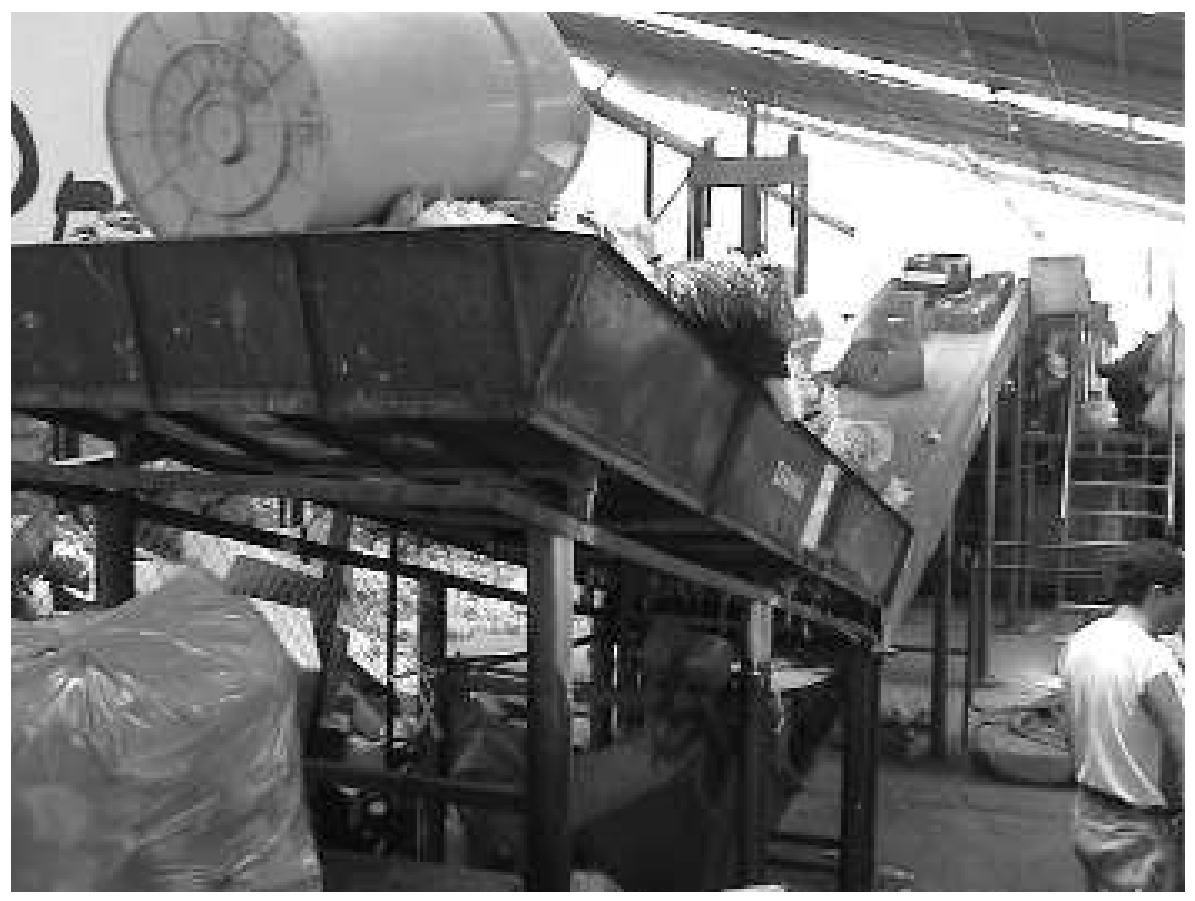

Elaboración propia. 
En la región Cusco, la mayor cantidad de generación de residuos sólidos proviene de la ciudad del Cusco y supone más del $60 \%$ del total de producción, cuya caracterización se compone principalmente de materia orgánica, además de los componentes de plásticos, papel, cartón, vidrio, metales, textil, madera y otros.

En el gráfico 2 se puede apreciar la distribución porcentual a escala provincial de los residuos sólidos, correspondiendo a la provincia del Cusco un $66 \%$, seguida por Canchis y Urubamba con un $13 \%$ y $8 \%$, respectivamente, y Santa Teresa, que pertenece a la provincia de La Convención, participa con un $2 \%$.

\section{Gráfico 2}

Distribución de residuos sólidos por provincias

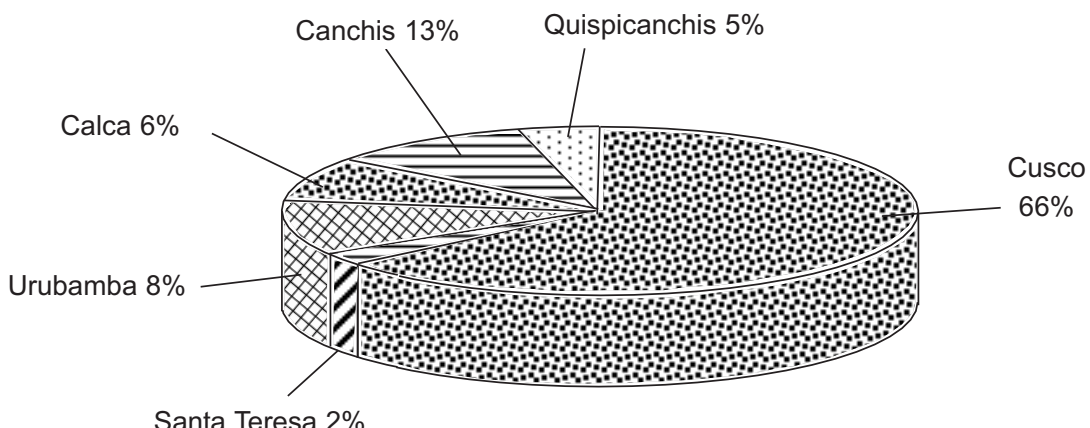

Santa Teresa $2 \%$

Elaboración propia.

En el cuadro 1 se presenta la producción de residuos sólidos de los diferentes distritos a lo largo de la cuenca del río Vilcanota, donde se puede apreciar que la mayor producción corresponde a Cusco capital con sus distritos San Sebastián, Santiago, Wanchaq y San Jerónimo, seguida por Sicuani, que pertenece a la provincia de Canchis. 


\section{Cuadro 1}

Producción de residuos sólidos por distritos - Cusco

\begin{tabular}{|c|c|c|c|}
\hline Distritos & $\begin{array}{l}\text { Población } \\
\text { total } \\
2007\end{array}$ & $\begin{array}{l}\text { Producción de } \\
\text { residuos sólidos } \\
\text { (t/día) }\end{array}$ & $\begin{array}{l}\text { Producción de } \\
\text { residuos sólidos } \\
\text { (t/año) }\end{array}$ \\
\hline Machu Picchu & 3.626 & 2,07 & 754 \\
\hline Ollantaytambo & 10.122 & 5,67 & 2.069 \\
\hline Maras & 7.122 & 3,35 & 1.222 \\
\hline Urubamba & 19.124 & 11,47 & 4.188 \\
\hline Yucay & 3.042 & 1,28 & 466 \\
\hline Huayllabamba & 5.260 & 2,16 & 787 \\
\hline Chincheros & 10.093 & 4,95 & 1.805 \\
\hline Calca & 19.155 & 10,15 & 3.706 \\
\hline Lamay & 5.737 & 2,81 & 1.026 \\
\hline Ccoya & 3.747 & 1,84 & 670 \\
\hline Pisac & 9.316 & 4,84 & 1.768 \\
\hline San Salvador & 4.982 & 2,44 & 891 \\
\hline Poroy & 4.930 & 2,22 & 810 \\
\hline Ccorca & 2.418 & 1,09 & 397 \\
\hline Santiago & 65.135 & 39,73 & 14.502 \\
\hline Cusco & 105.611 & 70,76 & 25.827 \\
\hline Wanchaq & 55.014 & 35,76 & 13.052 \\
\hline San Sebastián & 94.362 & 57,56 & 21.010 \\
\hline San Jerónimo & 31.138 & 16,19 & 5.910 \\
\hline Saylla & 2.915 & 1,46 & 532 \\
\hline Oropesa & 6.342 & 3,17 & 1.157 \\
\hline Andahuaylillas & 5.575 & 2,62 & 956 \\
\hline Huaro & 4.615 & 2,17 & 792 \\
\hline Urcos & 10.069 & 5,84 & 2.132 \\
\hline Quiquijana & 10.289 & 4,84 & 1.765 \\
\hline Cusipata & 4.351 & 2,04 & 746 \\
\hline Pitumarca & 8.199 & 3,85 & 1.407 \\
\hline Checacupe & 5.752 & 2,70 & 987 \\
\hline Combapata & 5.128 & 2,41 & 880 \\
\hline Tinta & 6.218 & 2,61 & 953 \\
\hline San Pedro & 3.175 & 1,33 & 487 \\
\hline San Pablo & 5.942 & 2,50 & 911 \\
\hline Sicuani & 58.519 & 30,43 & 11.107 \\
\hline Maranganí & 12.542 & 5,27 & 1.923 \\
\hline Total & 609.565 & 347 & 127.785 \\
\hline
\end{tabular}

Fuente: Imaitec Consultores. Lima, 2007. 


\section{LOS RESIDUOS SÓLIDOS Y SUS EFECTOS SOBRE EL MEDIO AMBIENTE}

Se estima que entre el $40 \%$ y el $50 \%$ del total de residuos sólidos generados en la cuenca del Vilcanota son vertidos en las márgenes de los ríos, carreteras, canales de aguas y chacras, entre otros. Es decir que el vertido de residuos (véase fotografía 3) se realiza principalmente en el cauce del río Vilcanota, por los pueblos ubicados en la parte alta de la cuenca, comenzando en La Raya y pasando por el distrito de Maranganí, en Sicuani, hasta Machu Picchu pueblo, incluyendo Santa Teresa, cuya cuenca tiene una longitud aproximada de 400 kilómetros.

El vertido incontrolado de los residuos sólidos en el valle del Vilcanota ocasiona serios impactos ambientales que afectan en especial el agua, el suelo y la salud humana y, en general, provocan el deterioro de los ecosistemas naturales y ponen en grave riesgo el aprovechamiento de los atributos turísticos de la región; por ello, la gestión y el manejo adecuado de los residuos sólidos en la cuenca del Vilcanota contribuirá a mejorar la calidad ambiental, mediante la conservación y protección de las áreas naturales y los lugares históricos y arqueológicos, y brindará a los turistas un lugar atractivo y descontaminado.

\section{Fotografía 3}

\section{Vertido de residuos sólidos en las márgenes del río Vilcanota}

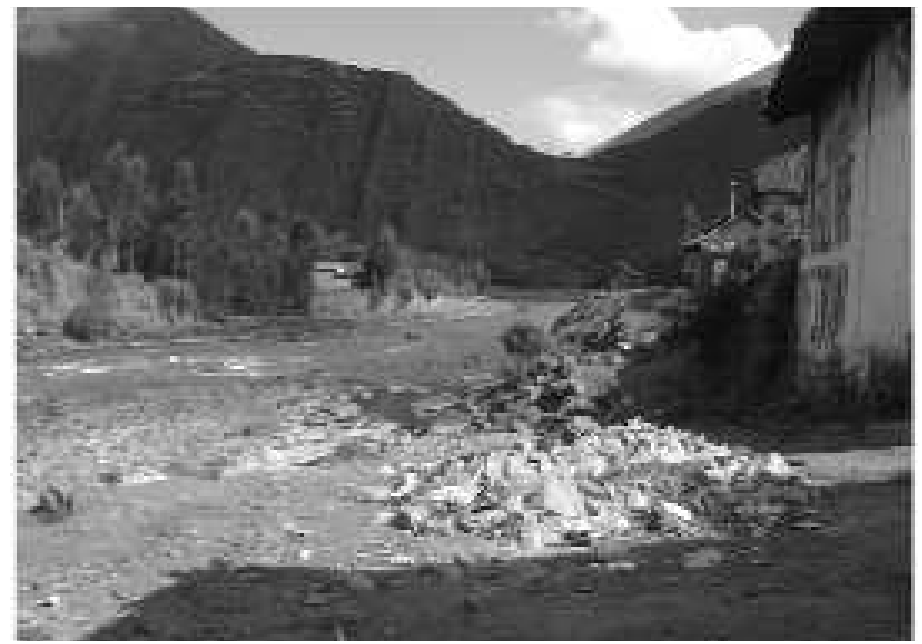

Elaboración propia. 


\section{EL TURISMO EN LA REGIÓN CUSCO Y EL MEDIO AMBIENTE}

En los cuadros 2 y 3 se puede apreciar la cantidad de visitantes extranjeros y nacionales que llegan al Perú, y principalmente al santuario de Machu Picchu. Es importante notar que la zona de mayor afluencia de turistas se concentra en la ciudad de Cusco y en Pisac, Calca, Urubamba, Ollantaytambo y Machu Picchu, y en la ruta Caminos del Inca, donde se realiza turismo rural.

El Valle Sagrado de los Incas recibe una gran cantidad de turistas debido a la ubicación de importantes monumentos arqueológicos, registrándose un incremento del 80\% entre los años 2001 (420.871 visitantes) y 2007 (737.576 turistas). Asimismo, se puede observar que el número de visitantes que llegó al Perú fue de 997.628 turistas en el 2002 y de 1.812 .384 turistas el 2007 , cuyo crecimiento durante este periodo se acercó al 50\%.

En la actualidad hay un gran interés de parte de diversos organismos nacionales e internacionales no solo en realizar estudios de gestión y manejo integral de los residuos sólidos en el valle del Vilcanota, incluyendo la posibilidad de realizar estudios de Desarrollo de Mecanismo Limpio (MDL), según lo establecido en el Tratado de Kyoto, sino que además contempla estudios de descontaminación integral de la cuenca del Vilcanota, para evitar el deterioro del medio natural y con la finalidad de hacerla sostenible ambiental, económica y socialmente.

Con el objetivo de plantear proyectos de descontaminación integral de la cuenca, existen diversas competencias y ciertas confusiones, por ejemplo, en la gestión y manejo de residuos sólidos en lo que concierne a la jurisdicción del distrito de Machu Picchu, la municipalidad correspondiente es la responsable de la gestión; sin embargo, el santuario histórico de Machu Picchu y los parques arqueológicos son responsabilidad del Instituto Nacional de Cultura (INC) en el manejo de residuos sólidos; del mismo modo, la ruta Caminos del Inca así como los campamentos, son responsabilidad del Instituto Nacional de Recursos Naturales (Inrena). También están las competencias de los alcaldes provinciales y distritales a lo largo de la cuenca del Vilcanota. 


\section{Cuadro 2}

Visitantes al Perú de turistas extranjeros no residentes

\begin{tabular}{lrrrrrrrr}
\hline Mes & $\mathbf{2 0 0 2}$ & $\mathbf{2 0 0 3}$ & $\mathbf{2 0 0 4}$ & $\mathbf{2 0 0 5}$ & $\mathbf{2 0 0 6} \mathbf{P}$ & $\mathbf{2 0 0 7} \mathbf{P}$ & $\begin{array}{r}\text { Var. \% } \\
\mathbf{0 6 / 0 5}\end{array}$ & $\begin{array}{r}\text { Var. \% } \\
\mathbf{0 7 / 0 6}\end{array}$ \\
\hline Enero & & & & & & & & \\
Febrero & 81.451 & 83.897 & 99.631 & 117.299 & 134.167 & 146.761 & 14,4 & 9,4 \\
Marzo & 80.838 & 84.396 & 103.100 & 119.207 & 140.711 & 150.842 & 18,0 & 7,2 \\
Abril & 80.986 & 79.796 & 97.422 & 117.883 & 133.936 & 150.220 & 13,6 & 12,2 \\
Mayo & 78.198 & 75.358 & 92.825 & 106.977 & 128.006 & 132.263 & 19,7 & 3,3 \\
Junio & 80.889 & 85.687 & 103.359 & 115.102 & 128.970 & 146.447 & 12,0 & 13,6 \\
Julio & 101.022 & 112.259 & 137.313 & 150.156 & 159.916 & 179.901 & 6,5 & 12,5 \\
Agosto & 92.711 & 103.683 & 114.252 & 138.564 & 143.089 & 159.368 & 3,3 & 11,4 \\
Setiembre & 80.041 & 87.240 & 100.636 & 124.549 & 132.223 & 146.760 & 6,2 & 11,0 \\
Octubre & 82.327 & 86.748 & 109.098 & 127.149 & 134.191 & 150.202 & 5,5 & 11,9 \\
Noviembre & 80.862 & 90.501 & 105.484 & 120.209 & 130.529 & 152.727 & 8,6 & 17,0 \\
Diciembre & 89.179 & 101.768 & 121.068 & 138.147 & 144.463 & 164.190 & 4,6 & 13,7 \\
\hline TOTAL & 997.628 & 1.069 .517 & 1.276 .639 & 1.486 .502 & 1.634 .745 & 1.812 .384 & 10,0 & 10,9
\end{tabular}

P: cifra preliminar

Fuente: Mincetur, 2008.

\section{Cuadro 3}

\section{Visitantes extranjeros y nacionales al santuario histórico de Machu Picchu}

\begin{tabular}{lccccccccccc}
\hline Año/mes & $\mathbf{1 9 9 7}$ & $\mathbf{1 9 9 8}$ & $\mathbf{1 9 9 9}$ & $\mathbf{2 0 0 0}$ & $\mathbf{2 0 0 1}$ & $\mathbf{2 0 0 2}$ & $\mathbf{2 0 0 3}$ & $\mathbf{2 0 0 4}$ & $\mathbf{2 0 0 5}$ & $\mathbf{2 0 0 6}$ & $\mathbf{2 0 0 7}$ \\
\hline Enero & 25.200 & 26.738 & 28.159 & $\mathbf{3 2 . 8 9 9}$ & $\mathbf{3 0 . 5 8 7}$ & 29.916 & 29.832 & 35.574 & 46.793 & 50.845 & 55.712 \\
Febrero & 20.358 & 19.960 & 22.205 & 29.483 & 26.602 & 21.121 & 20.349 & 25.259 & 32.172 & 34.130 & 39.105 \\
Marzo & 19.159 & 15.081 & 24.117 & 28.625 & 25.005 & 29.129 & 28.696 & 34.274 & 50.877 & 49.337 & 55.092 \\
Abril & 16.653 & 19.170 & 24.127 & 29.321 & 29.546 & 28.080 & 34.862 & 41.232 & 43.241 & 52.734 & 59.003 \\
Mayo & 19.256 & 21.787 & 25.278 & 29.340 & 31.214 & 34.711 & 37.670 & 44.455 & 53.641 & 55.001 & 60.504 \\
Junio & 18.102 & 23.670 & 27.141 & 31.184 & 32.233 & 33.829 & 38.951 & 44.999 & 51.977 & 50.019 & 58.842 \\
Julio & 31.533 & 36.454 & 43.357 & 44.294 & 45.409 & 46.963 & 55.486 & 61.028 & 69.960 & 66.153 & 75.737 \\
Agosto & 36.062 & 42.537 & 49.665 & 52.201 & 50.774 & 57.613 & 61.737 & 69.801 & 78.550 & 77.732 & 89.380 \\
Setiembre & 20.324 & 26.120 & 31.131 & 36.940 & 36.609 & 41.053 & 47.124 & 50.136 & 59.623 & 61.252 & 67.888 \\
Octubre & 33.664 & 36.907 & 41546 & 43111 & 45.443 & 52.722 & 63.101 & 71.456 & 75.308 & 76.651 & 90.518 \\
Noviembre & 30.303 & 37.790 & 31897 & 35697 & 40.677 & 50.625 & 61.481 & 59.980 & 69.212 & 66.158 & 85.795 \\
Diciembre & 23.418 & 28.349 & 25162 & 27295 & 26.772 & 33.490 & 44.519 & 49.169 & 48.599 & 51.611 & 84.350 \\
\hline Total & 294.032 & 334.563 & 373.785 & 420.390 & 420.871 & 459.252 & 523.808 & 587.363 & 679.953 & 691.623737 .576
\end{tabular}

Fuente: Mincetur, 2008. 
En el cuadro 4 se puede apreciar que la generación de residuos sólidos en la ruta Caminos del Inca es estacionaria, registrándose la mayor cantidad de residuos durante los meses de julio, agosto y setiembre, lo cual representa un aumento del 13\% anual. Es decir, estos son los meses considerados de temporada alta en la actividad turística en la región Cusco.

Por otra parte, los residuos sólidos generados por los turistas en los campamentos durante el recorrido de la ruta Caminos del Inca, como se puede ver en la fotografía 4, son almacenados en diferentes centros de acopio para su posterior traslado por medio de Perú Rail hasta la estación de transferencia denominada Pachar, en Ollantaytambo, y de ahí, mediante camiones, hasta el vertido en una cantera cercana a Ollantaytambo. Hasta mayo del año pasado la disposición de residuos se realizaba en el relleno sanitario de Yuncachahuayco, fecha en que fue clausurado por oposición de los comuneros de la zona, debido a posibles impactos producidos sobre la agricultura circundante.

\section{Fotografía 4}

Punto de acopio de residuos sólidos en la ruta Caminos del Inca

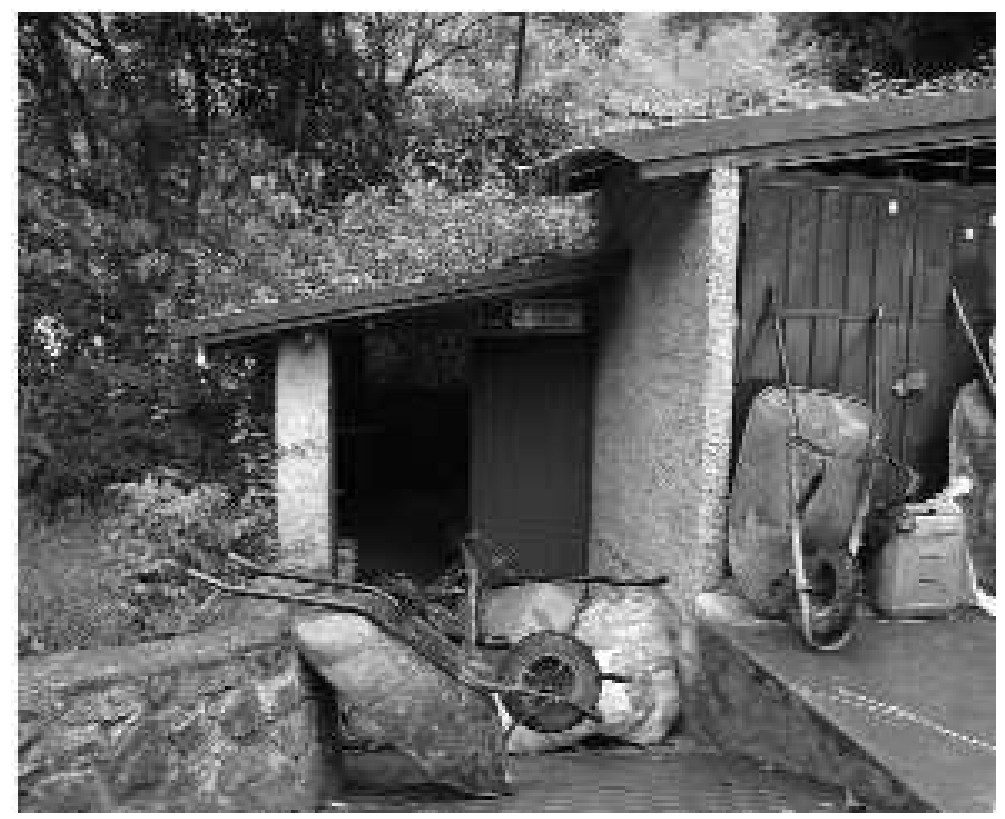

Elaboración propia. 


\section{Cuadro 4}

Generación de residuos sólidos en la ruta Caminos del Inca

\begin{tabular}{lrr}
\hline Meses & $\mathbf{K g}$ & $\mathbf{\%}$ \\
\hline Enero & 3.479 & 4,15 \\
Febrero & 1.234 & 1,47 \\
Marzo & 3.110 & 3,71 \\
Abril & 3.855 & 4,60 \\
Mayo & 5.907 & 7,05 \\
Junio & 8.067 & 9,63 \\
Julio & 11.914 & 14,11 \\
Agosto & 12.056 & 14,40 \\
Setiembre & 11.187 & 13,36 \\
Octubre & 10.051 & 12,00 \\
Noviembre & 7.190 & 8,59 \\
Diciembre & 5.797 & 6,92 \\
\hline Total & 83.747 & 100,00 \\
\hline
\end{tabular}

Fuente: Selip Cusco, 2006.

\section{BIBLIOGRAFÍA}

Instituto Tecnológico Geominero de España. "Gestión y manejo integral de residuos sólidos en España”. Madrid, 2001.

Imaitec Consultores. "Estudio de residuos sólidos en el valle del Vilcanota". Lima, 2007.

Mincetur. "Sistema de información de estadística de turismo". Lima, 2007.

Mincetur. "Plan de reordenamiento y rehabilitación del valle del Vilcanota". Guía marco para la evaluación ambiental de obras de infraestructura menor. Lima, 2007.

Servicio de Limpieza Pública del Cusco. "Plan integral de manejo de residuos sólidos en la ciudad del Cusco". Cusco, 2004. 